\section{Conhecimentos e atitudes da população sobre dengue no Município de São Luís, Maranhão, Brasil, 2004}

\author{
Public knowledge and attitudes concerning \\ dengue in the Municipality of São Luís, \\ Maranhão, Brasil, 2004
}

Vicente Silva Gonçalves Neto 1 Silvio Gomes Monteiro ${ }^{2}$ Azizedite Guedes Gonçalves 3 José Manuel Macário Rebêlo 2

\author{
1 Agência Nacional \\ de Vigilância Sanitária \\ São Luís, Brasil. \\ 2 Departamento de Biologia, \\ Universidade Federal do \\ Maranhão, São Luís, Brasil. \\ 3 Departamento de \\ Patologia, Universidade \\ Federal do Maranhão, \\ São Luís, Brasil. \\ Correspondência \\ V. S. Gonçalves Neto \\ Posto Aeroportuário, \\ Agência Nacional de \\ Vigilância Sanitária. \\ Av. dos Libaneses $s / n$, \\ São Luís, MA \\ 65025-560, Brasil. \\ vicente.neto@anvisa.gov.br
}

\begin{abstract}
A sample survey with 843 interviews in São Luís, Maranhão State, Brazil was conducted to assess community knowledge and concepts regarding dengue. The population's understanding and its socioeconomic, health, and environmental conditions were evaluated. Potential vector breeding sites were found in all the areas surveyed. Media (TV, radio, etc.) were the most important source of public information on the disease. However, a gap was observed between knowledge and attitudes towards vector prevention. Strategies are also needed to involve the population in more effective prevention of vector proliferation, thus helping control the disease in São Luís.
\end{abstract}

Dengue; Aedes; Health Knowledge, Attitudes, Practice

\section{Introdução}

O dengue constitui atualmente a mais importante arbovirose transmitida por insetos no que se refere à morbi-mortalidade. Atinge em torno de 100 países das regiões tropicais e subtropicais 1 .

Os mosquitos vetores da doença pertencem ao gênero Aedes. O Ae. aegypti, também transmissor da febre amarela urbana, é o mais importante e encontra-se disperso no continente americano desde o sul dos Estados Unidos até o Uruguai 1 . Outro mosquito que merece atenção é o Ae. albopictus, que apesar de ainda não ter comprovado o seu papel como vetor do vírus do dengue no continente americano, é citado por Silveira 2 como elo importante na cadeia de transmissão da doença na Ásia.

No Estado do Maranhão, notificou-se de 1982 a 1997, 14.190 casos da doença 2 e 39.764 casos nos anos de 1998 a 2002 1,3,4.

No Município de São Luís, capital do Estado do Maranhão, foram notificados 12.008 casos no intervalo de tempo de 1997 a 20025.

Na ilha de São Luís, dados da Fundação Nacional de Saúde (FUNASA) do ano de 1995 destacam a grande infestação do Ae. aegypti e a facilidade de sua dispersão para outras áreas do estado devido ao constante fluxo de migração interna 6 .

O controle da transmissão do vírus do dengue acontece essencialmente no âmbito coletivo e exige um esforço de toda a sociedade, em 
virtude da elevada capacidade de adaptação e dispersão do Ae. aegypti. Assim, a população dessas áreas com ocorrência de transmissão necessita adquirir informações que visem a mudanças de atitudes que possam ajudar na prevenção e no controle dessa doença.

As precárias condições de infra-estrutura sanitária e a explosão demográfica em áreas tropicais despontam como requisitos importantes para a proliferação do vetor e conseqüente transmissão da doença 6 . Além do mais, as concentrações de população de baixa renda em áreas periféricas das grandes cidades criam ambiente favorável ao desenvolvimento e proliferação do Ae. aegypti, com criadouros disponibilizados pelo homem como conseqüência da precariedade nos serviços de coleta de lixo e abastecimento de água; daí a necessidade urgente de uma participação efetiva da sociedade na implementação de medidas efetivas de coleta de lixo, regularidade no abastecimento de água potável e harmonização do espaço público objetivando ao máximo a redução dos potenciais criadouros dos mosquitos 7 .

Em razão da complexidade e da extensão do problema "dengue", propôs-se realizar uma investigação junto à população do Município de São Luís para entender o conhecimento, suas atitudes e informações a respeito da transmissão, prevenção e controle deste agravo.

Nesse contexto, por meio deste estudo objetivou-se: avaliar informações dos entrevistados quanto ao conhecimento do dengue; descrever as condições sócio-econômicas, sanitárias e ambientais das comunidades; identificar os tipos de criadouros potenciais do mosquito e conhecer as atitudes das comunidades sobre as medidas de controle da doença.

\section{Material e métodos}

\section{Área de estudo}

O Município de São Luís está situado na ilha de São Luís, ao norte do Estado do Maranhão (2o 31 ' Sul e $44^{\circ} 18^{\prime}$ Oeste), altitude média de $32 \mathrm{~m}$ e área de $822,1 \mathrm{~km}^{2}$ que corresponde aproximadamente $0,24 \%$ do território do estado.

O clima é o tropical quente e úmido, com duas estações: a chuvosa (janeiro a junho), com precipitação pluviométrica média de $1.954 \mathrm{~mm}$; e a de estiagem (julho a dezembro). A temperatura varia entre 28 a $30^{\circ} \mathrm{C}$, em média.

O Município de São Luís é cortado por diversos rios e estuários, entre eles pode-se citar: Anil, Bacanga, Tibiri, Mosquito, Mocajituba, Antônio Neves, São João e Paciência 8 .
Segundo o Instituto Brasileiro de Geografia e Estatística (IBGE) 8, em 2000 a população de São Luís era de 855.442 habitantes, residindo em 276 mil domicílios. O município está dividido administrativamente em sete distritos sanitários: (1) Centro, (2) Itaqui Bacanga, (3) Coroadinho, (4) Cohab, (5) Bequimão, (6) Tirirical e (7) Vila Esperança que apresentam em média, cada um, 50 localidades 9 .

\section{Tipo de estudo}

Realizou-se um estudo transversal com aplicação de um inquérito eco-epidemiológico aleatório, no período de abril a maio de 2004. A aleatorização foi feita utilizando o programa BioEstat versão 3.0 10. Definiu-se que seria obtida uma amostra estratificada por distrito sanitário, de 843 domicílios, considerando-se que a proporção média de afetados por imóvel em São Luís é de 0,04\% 11 e que em áreas mais críticas o número de afetados por imóveis seja dez vezes maior. Foi considerado como poder de teste $80 \%$ e o nível de significância igual a $5 \%$.

\section{Amostragem}

A amostragem foi estratificada em diferentes níveis baseando-se no número de habitantes dos distritos sanitários, e nestes foram selecionados aleatoriamente os bairros que teriam os domicílios investigados. Dentro de cada bairro, o número de domicílios investigados foi proporcional à população. Após o sorteio dos domicílios, tomou-se um croqui de cada bairro sorteado para localizar, por quadra, cada domicílio a ser visitado.

\section{Coleta de dados}

Os dados foram coletados usando-se um questionário previamente testado em um bairro da cidade, para confirmar a adequação do instrumento, visando à qualidade das informações obtidas. Tomou-se por base o domicílio como unidade amostral, entrevistando-se a pessoa responsável a partir de 18 anos. O domicílio sorteado encontrado fechado foi substituído pelo imediatamente a seguir. Realizou-se supervisão em $5 \%$ dos domicílios pesquisados com a finalidade de monitorar as informações obtidas.

O questionário aplicado contemplou dados como: identificação, situações socioeconômica e sanitário-ambiental, bem como medidas de prevenção.

A coleta dos dados foi feita por agentes de saúde que fazem o trabalho de campo do Programa de Controle da Febre Amarela e Dengue 
(PCFAD), da Secretaria Municipal de Saúde de São Luís (SEMUS). Após as devidas correções e adaptações foi elaborado o questionário definitivo e executado o inquérito domiciliar. $\mathrm{O}$ projeto de pesquisa foi aprovado pelo Comitê de Ética em Pesquisa da Universidade Federal do Maranhão. Foi assegurado o conhecimento e a concordância dos entrevistados, assim como a confidenciabilidade das informações.

Sobre criadouros potenciais foram considerados aqueles que continham água e que poderiam oferecer condições para a procriação dos mosquitos transmissores do dengue 12 .

A variável sintomas, por apresentar extenso número de manifestações 13, foi agrupada, variando de um a mais de três, citando-se os mais freqüentes: febre, dor no corpo (olhos, ossos), vômitos e manchas vermelhas pelo corpo.

\section{Análise dos dados}

Os dados foram armazenados e analisados no programa Epi Info 6.0 (Centers for Disease Control and Prevention, Atlanta, Estados Unidos). Inicialmente, foram procedidas análises de estatística descritiva dos dados (tabelas de freqüências) com intervalos de confiança de 95\%. Posteriormente, utilizou-se tabela de contingência cruzando as variáveis dependentes (respostas sobre o conhecimento da dengue, sobre os aspectos ambientais e sobre as atitudes preventivas) com as variáveis independentes (distrito, sexo, escolaridade e renda), que foram avaliadas pela análise de variância multivariada (MANOVA). Em todos os testes, o nível de significância utilizado para se rejeitar a hipótese de nulidade foi de $5 \%$.

\section{Resultados}

Do total de 843 residentes pesquisados, $75,2 \%$ $(72,5$ a $77,7 \%)$ eram do sexo feminino e $24,8 \%$ $(0,218$ a $0,272 \%)$ do sexo masculino, nas faixas etárias: $35,5 \%(0,324$ a $0,381 \%)$ de 18 a 30 anos; $52,4 \%(48,9$ a $55,2 \%)$ de 30 a 60 anos e $12,1 \%(9,9$ a 13,4\%) com mais de 60 anos. Em relação ao estado civil, $42,1 \%(38,7$ a $44,8 \%)$ eram casados e $41,9 \%$ (38,4 a $44,6 \%)$ solteiros.

A respeito das condições sócio-econômicas dos entrevistados (Tabela 1), verificou-se que os alfabetizados representaram $94,6 \%$. Constatou-se ainda que em $76,7 \%$ dos domicílios residiam de 2 a 6 pessoas e que a renda familiar de 82,7\% não ultrapassava três salários mínimos.

Em relação ao conhecimento sobre dengue (Tabela 2), a maior parte da população, 99,4\% e 93,5\%, respectivamente, já ouviram falar em dengue e dengue hemorrágica; do mesmo mo-
Tabela 1

Condições sócio-econômicas dos entrevistados a respeito de dengue.

Município de São Luís, Maranhão, Brasil, 2004.

\begin{tabular}{|c|c|c|c|}
\hline Variáveis & Freqüência & $\%$ & IC95\% \\
\hline \multicolumn{4}{|l|}{ Escolaridade } \\
\hline Fundamental completo & 172 & 20,4 & $17,6-23,1$ \\
\hline Fundamental incompleto & 204 & 24,2 & $21,2-26,5$ \\
\hline Médio completo & 337 & 40,0 & $37,1-43,3$ \\
\hline Médio incompleto & 47 & 5,6 & $4,2-6,8$ \\
\hline Superior completo & 26 & 3,0 & $2,0-3,9$ \\
\hline Superior incompleto & 12 & 1,4 & $0,6-2,0$ \\
\hline Analfabeto & 30 & 3,6 & $2,3-4,8$ \\
\hline Não responderam & 15 & 1,8 & $1,0-2,6$ \\
\hline \multicolumn{4}{|l|}{ Residentes por domicílio } \\
\hline Mora só & 16 & 1,9 & $1,0-2,5$ \\
\hline 2 a 3 pessoas & 286 & 34,0 & $30,5-36,5$ \\
\hline 4 a 6 pessoas & 360 & 42,7 & $38,8-45,1$ \\
\hline Mais de 4 pessoas & 174 & 20,6 & $17,4-22,8$ \\
\hline Não responderam & 7 & 0,8 & $5,5-10,1$ \\
\hline \multicolumn{4}{|c|}{ Renda familiar (salários mínimos) } \\
\hline$<1$ & 241 & 28,6 & $25,8-31,3$ \\
\hline $1-3$ & 456 & 54,1 & $51,3-56,7$ \\
\hline $4-6$ & 65 & 7,7 & $5,9-9,3$ \\
\hline $7-10$ & 26 & 3,1 & $1,9-4,0$ \\
\hline+10 & 27 & 3,2 & $2,1-4,2$ \\
\hline Não responderam & 28 & 3,3 & $2,3-4,3$ \\
\hline Total & 843 & 100,0 & \\
\hline
\end{tabular}

do, 78,3\% referiram que a doença é causada pela picada do Ae. aegypti. Sobre a forma hemorrágica da doença, $85,1 \%$ responderam "dengue na forma grave" (mais forte, com hemorragia). Ao informar os sintomas clássicos do dengue, $94 \%$ dos participantes referiram de dois a mais de três. Ao serem perguntados sobre a transmissão, 95\% da população investigada responderam que era por meio da picada de mosquito. Indagados sobre como é o transmissor da dengue, $78,3 \%$ da população responderam pernilongo ou mosquito rajado e $11,1 \%$ informaram somente pernilongo. A respeito dos locais de procriação do transmissor, $92 \%$ dos entrevistados identificaram a água limpa e parada como ideal para a colocação dos seus ovos.

Quando se questionou sobre as fontes das informações obtidas sobre a doença, 92,6\% da população pesquisada referiram a mídia (TV, rádio) como veículo mais freqüente.

Questionados sobre existência de casos de dengue no domicílio, 53,3\% responderam afir- 
Tabela 2

Conhecimento da população sobre dengue.

Município de São Luís, Maranhão, Brasil, 2004.

\begin{tabular}{|c|c|c|c|}
\hline Variáveis & Freqüência & $\%$ & IC95\% \\
\hline \multicolumn{4}{|l|}{ Você já ouviu falar em dengue? } \\
\hline Sim & 838 & 99,4 & $98,8-99,9$ \\
\hline Não & 5 & 0,6 & $0,2-0,8$ \\
\hline \multicolumn{4}{|l|}{$\begin{array}{l}\text { Você já ouviu falar em dengue } \\
\text { hemorrágica? }\end{array}$} \\
\hline Sim & 788 & 93,5 & $91,6-94,9$ \\
\hline Não & 55 & 6,5 & $5,0-7,7$ \\
\hline \multicolumn{4}{|l|}{ O que é dengue? } \\
\hline $\begin{array}{l}\text { Doença causada por picada } \\
\text { do Aedes aegypti }\end{array}$ & 660 & 78,3 & $75,3-80,6$ \\
\hline $\begin{array}{l}\text { Doença causada por picada } \\
\text { de mosquito }\end{array}$ & 135 & 16,0 & $13,8-17,6$ \\
\hline Não sabe & 36 & 4,3 & $3,1-5,7$ \\
\hline Outras respostas & 12 & 1,4 & $0,7-1,9$ \\
\hline \multicolumn{4}{|l|}{ O que é dengue hemorrágica? } \\
\hline Dengue na forma grave & 717 & 85,1 & $82,5-87,0$ \\
\hline Não sabe & 117 & 13,9 & $11,8-15,8$ \\
\hline Outras respostas & 9 & 1,0 & $0,4-1,6$ \\
\hline \multicolumn{4}{|l|}{ Quais os sintomas do dengue? } \\
\hline Um sintoma & 40 & 4,7 & $3,2-5,9$ \\
\hline Dois sintomas & 247 & 29,3 & $26,3-32,0$ \\
\hline Três sintomas & 227 & 27,0 & $24,6-29,7$ \\
\hline Mais de três & 318 & 37,7 & $34,5-40,5$ \\
\hline Não responderam & 11 & 1,3 & $0,5-1,8$ \\
\hline \multicolumn{4}{|l|}{ Quais as formas de transmissão? } \\
\hline Picada de mosquito & 801 & 95,0 & $93,5-96,1$ \\
\hline Outras & 37 & 4,4 & $3,0-5,6$ \\
\hline Não sabiam & 5 & 0,6 & $0,1-1,1$ \\
\hline \multicolumn{4}{|l|}{ Como é o transmissor do dengue? } \\
\hline Pernilongo ou mosquito rajado & 660 & 78,3 & $75,3-80,6$ \\
\hline Pernilongo & 94 & 11,1 & $9,0-12,8$ \\
\hline Não sabiam & 89 & 10,6 & $8,8-12,2$ \\
\hline \multicolumn{4}{|c|}{ Onde se cria o mosquito transmissor? } \\
\hline Locais com água parada e limpa & 776 & 92,0 & $89,9-93,6$ \\
\hline Qualquer lugar & 49 & 5,8 & $4,3-7,2$ \\
\hline Outros & 18 & 2,2 & $1,4-3,0$ \\
\hline \multicolumn{4}{|c|}{ Onde você obteve essas informações? } \\
\hline Mídia (TV, rádio, jornal etc.) & 781 & 92,6 & $90,6-94,0$ \\
\hline Agente de saúde pública & 39 & 4,6 & $3,2-5,8$ \\
\hline Profissional de saúde & 4 & 0,5 & $0,1-0,8$ \\
\hline Outros & 19 & 2,3 & $1,6-3,0$ \\
\hline \multicolumn{4}{|l|}{$\begin{array}{l}\text { Alguém no domicílio já teve } \\
\text { ou está com dengue? }\end{array}$} \\
\hline Sim & 449 & 53,3 & $50,2-56,1$ \\
\hline Não & 383 & 45,4 & $41,3-48,3$ \\
\hline Não sabiam & 11 & 1,3 & $0,5-1,9$ \\
\hline \multicolumn{4}{|l|}{ Procurou tratamento? } \\
\hline Sim & 337 & 40,0 & $36,5-43,2$ \\
\hline Não & 506 & 60,0 & $56,2-63,1$ \\
\hline \multicolumn{4}{|l|}{ Onde buscou tratamento? } \\
\hline Unidade de Saúde & 158 & 18,7 & $16,3-20,9$ \\
\hline Auto-atendimento & 685 & 81,3 & $78,1-83,2$ \\
\hline Total & 843 & 100,0 & \\
\hline
\end{tabular}

mativamente. Com relação à assistência médica, somente $40 \%$ da população a procurou ao constatar os sintomas. Como local de tratamento, $18,7 \%$ dos entrevistados procuraram uma Unidade de Saúde, os demais procederam automedicação.

As informações a respeito das condições sanitárias e ambientais da população podem ser observadas na Tabela 3.

Em relação aos criadouros potenciais encontrados (1.221), aqueles relacionados ao armazenamento de água, como tambor, tanque, barril, tina, tonel, depósito de barro e caixa d'água foram os mais freqüentes $(53,72 \%)$, seguidos de vasos com água e pratos de plantas $(10,89 \%)$, garrafa, lata, plástico $(10,4 \%)$, pneus $(9,17 \%)$, poço, cisterna, cacimba $(5,73 \%)$, recipiente natural $(2,73 \%)$, materiais de construção, peça de carro $(2,12 \%)$ e outros $(5,57 \%)$.

Na Tabela 4, estão apresentadas as respostas às indagações a respeito das atitudes das comunidades pesquisadas sobre o que fazer para evitar o mosquito do dengue, indicando porcentual de respostas corretas bastante significantes.

O resultado da MANOVA das informações sobre conhecimentos, aspectos ambientais e atitudes preventivas da população pesquisada, com determinadas variáveis independentes (distrito, sexo, escolaridade, renda e as interações duas a duas), não apresentou diferenças significativas $(p>0,05)$ para sexo e a maioria das interações. Entretanto, foi observado diferenças altamente significativas $(\mathrm{p}<0,05) \mathrm{em}$ relação a distrito, renda e escolaridade, e a interação entre renda e escolaridade.

A Tabela 5 apresenta a relação de significância pela MANOVA de cada variável dependente com as independentes. Com relação ao distrito sanitário, foi encontrada diferença significativa $(\mathrm{p}<0,05)$ na questão conhecimento sobre os sintomas e a fonte de informações. O mesmo foi observado para as variáveis ambientais, tais como abastecimento de água, destino dos dejetos e criadouros potenciais.

Quanto à escolaridade, verificou-se diferença significativa $(p<0,05)$ na questão conhecimento sobre os locais de procriação do transmissor e fonte de informações.

Quanto à influência da renda no conhecimento do dengue, só foi observada diferença significativa $(\mathrm{p}<0,05)$ no que se refere aos locais de procriação do vetor. Possivelmente, isso se deva à relação positiva que há entre renda familiar e grau de instrução, o que afetará o conhecimento mais aprofundado sobre a doença.

$\mathrm{Na}$ relação da renda com os aspectos ambientais, observou-se diferença significativa $(p<0,05)$ com criadouros potenciais. Verificou- 
se a ocorrência de estocagem em todas as classes de renda, com predomínio no uso de depósitos do tipo caixa d'água e cisterna nas classes de renda de 1 a mais de 10 salários mínimos, alcançando maior proporção nas classes de 7 a mais de 10; conseqüentemente, tais depósitos constituíram os principais criadouros. Entretanto, na classe de renda menor que um salário mínimo, predominou outros tipos de depósitos $(40,2 \%)$.

No que diz respeito às atitudes preventivas e a sua relação com a renda, observou-se uma dependência significativa $(p<0,05)$ em relação às seguintes atitudes: o que fazer para evitar $o$ mosquito do dengue em vasos e em pneus velhos, descartados no meio ambiente sem proteção?

O efeito significativo $(\mathrm{p}<0,05)$ da interação escolaridade e renda nas variáveis dependentes vetor, locais de procriação, fontes de informação e garrafas e latas, pode ser compreendido pelo fato delas apresentarem uma associação entre si, pois normalmente espera-se que quanto maior a escolaridade maior seja a renda e vice-versa.

De um modo geral, observou-se que o conhecimento sobre o dengue, o perfil sanitário e as atitudes sobre prevenção, são uniformes nos distritos sanitários, no entanto, Tirirical e Vila Esperança apresentaram níveis de escolaridade e de conhecimento mais baixos que os demais. Em relação ao perfil sanitário, encontrou-se também nesses distritos sanitários a mais baixa porcentagem de residências abastecidas de água pelo sistema público $(72,2 \% \mathrm{e}$ $51,2 \%$ ), respectivamente, e $26 \%$ e $41,8 \%$ servidas apenas de poço. Ressalta-se que ambos os distritos sanitários são proporcionalmente os que apresentam maior número de localidades rurais, onde os serviços públicos não estão disponibilizados na medida das suas necessidades.

Os distritos sanitários de Itaqui, Bequimão e Vila Esperança apresentaram baixa proporção na regularidade do serviço de abastecimento de água $(37,2 \%, 41,1 \%$ e $44,2 \%)$, respectivamente. Como conseqüência da intermitência no abastecimento, a estocagem de água é um procedimento comum nestes distritos sanitários.

Em relação aos dejetos, observou-se baixa porcentagem de residências ligadas ao sistema público de esgotamento sanitário, na ordem de $13,5 \%, 20,7 \%, 18,3 \%$ e $2,3 \%$, respectivamente, para os distritos sanitários de Itaqui, Cohab, Tirirical e Vila Esperança, e 70,5\%, 55,2\%, 74,8\% e $25,6 \%$ nesses mesmos distritos sanitários que se utilizam de fossas sépticas, chamando a atenção Vila Esperança e Cohab onde $67,4 \%$ e $24,1 \%$ das residências ainda utilizam-se de fossas negras.
Tabela 3

Condições sanitárias e ambientais da população entrevistada.

Município de São Luís, Maranhão, Brasil, 2004.

\begin{tabular}{|c|c|c|c|}
\hline Variáveis & Freqüência & $\%$ & IC95\% \\
\hline \multicolumn{4}{|c|}{ Origem da água para consumo } \\
\hline Abastecimento público & 729 & 86,5 & $83,9-88,1$ \\
\hline Poço & 79 & 9,4 & $7,2-10,9$ \\
\hline Outros & 35 & 4,1 & $2,9-5,2$ \\
\hline \multicolumn{4}{|c|}{ Abastecimento de água regular? } \\
\hline Sim & 473 & 56,1 & $53,0-58,7$ \\
\hline Não & 370 & 43,9 & $40,2-47,0$ \\
\hline \multicolumn{4}{|l|}{ Estocagem de água } \\
\hline Caixa d'água & 391 & 46,4 & $43,5-49,0$ \\
\hline Tonel & 129 & 15,3 & $12,8-17,3$ \\
\hline Outros & 254 & 30,1 & $26,7-33,2$ \\
\hline Não estoca & 69 & 8,2 & $6,2-9,4$ \\
\hline \multicolumn{4}{|l|}{ Esgotamento sanitário } \\
\hline Rede pública de coleta & 390 & 46,3 & $43,0-48,9$ \\
\hline Fossa séptica & 332 & 39,4 & $36,1-42,4$ \\
\hline Fossa negra & 86 & 10,2 & $8,1-12,2$ \\
\hline Outros & 35 & 4,1 & $2,7-5,2$ \\
\hline \multicolumn{4}{|l|}{ Destino do lixo doméstico } \\
\hline Coleta pública & 741 & 87,9 & $85,7-89,7$ \\
\hline Queimado & 64 & 7,6 & $5,8-9,1$ \\
\hline Terreno baldio & 15 & 1,8 & $1,0-2,5$ \\
\hline Outros & 23 & 2,7 & $1,8-3,5$ \\
\hline Total & 843 & 100,0 & \\
\hline
\end{tabular}

A coleta de lixo é realizada em $87,9 \%$ dos distritos sanitários, pelo sistema público, porém em Vila Esperança, constatou-se $69,8 \%$ das residências adotando o processo de queima dos resíduos.

Em relação a criadouros potenciais, encontrou-se $66 \%$ do tipo reservatórios permanentes para armazenamento de água (caixa d'água, barril, tambor, cisterna).

No geral, a renda de 1 a 3 salários mínimos é preponderante nos vários distritos sanitários. No entanto, Vila Esperança destaca-se com renda familiar menor que 1 salário mínimo em $67,4 \%$ das famílias.

Em relação à prevenção ao mosquito transmissor do dengue, observou-se que $84,6 \%$ da população entrevistada conhece as atitudes corretas para tais medidas. 
População entrevistada (843) segundo tipo de atitudes corretas sobre o que fazer para evitar o mosquito do dengue em vários tipos de criadouros. Município de São Luís, Maranhão, Brasil, 2004.

\begin{tabular}{|c|c|c|c|}
\hline Variáveis & Freqüência & $\%$ & IC95\% \\
\hline \multicolumn{4}{|l|}{ O que fazer para evitar que o mosquito do dengue se crie em vasos com água e pratos de plantas? } \\
\hline Atitudes corretas (trocar água e lavar com bucha 2 vezes/semana; colocar areia grossa no prato) & 631 & 74,9 & $72,4-76,9$ \\
\hline Parcialmente correta (alguma(s) das atitudes corretas) & 152 & 18,0 & $15,7-20,4$ \\
\hline Não sabe & 59 & 7,1 & $5,7-8,3$ \\
\hline \multicolumn{4}{|l|}{ O que fazer para evitar que o mosquito do dengue se crie em caixa d'água, barril, tambor, cisterna? } \\
\hline $\begin{array}{l}\text { Atitudes corretas (mantê-los bem tampados, sem frestas para impedir a entrada do vetor; } \\
\text { lavar bem antes de enchê-los) }\end{array}$ & 727 & 86,2 & $83,8-88,0$ \\
\hline Parcialmente correta (alguma(s) das atitudes corretas) & 93 & 11,0 & $9,1-12,9$ \\
\hline Não sabe & 23 & 2,8 & $1,8-3,7$ \\
\hline \multicolumn{4}{|l|}{ O que fazer para evitar que o mosquito do dengue se crie em pneus usados? } \\
\hline Atitudes corretas (cobrir, mantê-los secos, eliminar) & 702 & 83,3 & $80,6-85,4$ \\
\hline Parcialmente correta (alguma(s) das atitudes corretas) & 84 & 10,0 & $8,1-11,8$ \\
\hline Não sabe & 57 & 6,7 & $5,0-8,1$ \\
\hline \multicolumn{4}{|l|}{ O que fazer para evitar que o mosquito do dengue se crie em garrafas e latas? } \\
\hline Atitudes corretas (guardar de boca para baixo, eliminar, cobrir) & 795 & 94,3 & $92,9-95,3$ \\
\hline Parcialmente correta (alguma(s) das atitudes corretas) & 24 & 2,8 & $1,6-3,7$ \\
\hline Não sabe & 30 & 2,9 & $1,9-3,8$ \\
\hline Total & 843 & 100,0 & \\
\hline
\end{tabular}

\section{Discussão}

Considerando-se os resultados deste estudo, observa-se que de modo geral há uma boa parcela da população com conhecimentos sobre o dengue e o seu vetor, concordando com estudos semelhantes de Chiaravalloti Neto 14, Chiaravalloti Neto et al. 15 e Donalisio et al. 16. Essas informações foram adquiridas por meio da mídia e de campanhas educativas institucionais, incrementadas pelas informações repassadas pelo pessoal de campo do programa de controle do dengue, realização de palestras, distribuição de mensagens e cartazes, e efetivação de atividades com grupos variados (associação de bairro, clube de mães, grupos de idosos e de jovens etc.). Tais resultados corroboram aqueles obtidos por outros autores 14,16,17,18,19,20.

Apesar das informações adquiridas e considerando que a maioria dos entrevistados possuem pelo menos o ensino fundamental completo, portanto, com condições de apreender noções básicas de educação em saúde sobre dengue, a doença continua em expansão.

Não obstante ao nível de conhecimentos, constatou-se a existência de grande quantidade de criadouros de Aedes nas residências, fato que pode influenciar na ocorrência de epidemias de dengue, principalmente nos períodos chuvosos, indicando que a população mesmo conhecedora do ambiente onde se desenvolvem os mosquitos, pouco participa no sentido de atuar na redução desses locais de proliferação do inseto, à semelhança dos achados de Teixeira et al. 21,22. Isso se observa pelo aumento da utilização de materiais não recicláveis e sua disposição inadequada nas próprias residências e terrenos baldios, criando ambientes favoráveis para o desenvolvimento da fase aquática dos mosquitos 23,24 , tornando-se importante fator de risco para o surgimento de casos potenciais de dengue.

Em relação ao abastecimento de água, detectou-se que a maioria da população dispunha de água do sistema público, entretanto, a descontinuidade no abastecimento pelo sistema público desencadeia a estocagem pela população, que não confia na regularidade do serviço. Em muitas ocasiões a água somente chega nas torneiras no período da madrugada, obrigando a população a estocar em depósitos de grande porte. Nesse contexto, a caixa d'água como criadouro merece atenção especial, uma vez que é encontrada em uma parcela significativa 
Resultado da análise de variância multivariada das variáveis sobre conhecimentos, aspectos ambientais e atitudes da população pesquisada em relação às variáveis distrito sanitário, sexo, escolaridade e renda, e interação escolaridade e renda frente à situação do dengue. Município de São Luís, Maranhão, Brasil, 2004.

\begin{tabular}{|c|c|c|c|c|c|c|c|c|c|c|}
\hline \multirow[t]{2}{*}{ Variáveis } & \multicolumn{2}{|c|}{ Distrito sanitário } & \multicolumn{2}{|c|}{ Sexo } & \multicolumn{2}{|c|}{ Escolaridade } & \multicolumn{2}{|c|}{ Renda } & \multicolumn{2}{|c|}{$\begin{array}{l}\text { Interação escola } \\
\text { ridade e renda }\end{array}$} \\
\hline & Freqüência & $p$ & Freqüência & $\mathrm{p}$ & Freqüência & $\mathrm{p}$ & Freqüência & $p$ & Freqüência & $\mathrm{p}$ \\
\hline \multicolumn{11}{|l|}{ Conhecimentos } \\
\hline O que é dengue? & 1,423 & 0,203 & 0,226 & 0,635 & 0,75 & 0,630 & 0,168 & 0,974 & 0,846 & 0,677 \\
\hline O que é dengue hemorrágico? & 0,956 & 0,454 & 0,456 & 0,500 & 0,72 & 0,655 & 0,166 & 0,975 & 0,313 & 0,999 \\
\hline Quais os sintomas? & 2,663 & 0,015 & 1,142 & 0,286 & 1,134 & 0,340 & 2,196 & 0,053 & 0,805 & 0,732 \\
\hline Formas de transmissão & 0,125 & 0,993 & 0,523 & 0,470 & 0,216 & 0,982 & 0,016 & 1,000 & 0,190 & 1,000 \\
\hline Como é o vetor? & 1,983 & 0,066 & 0,284 & 0,594 & 0,971 & 0,451 & 0,305 & 0,910 & 2,626 & 0,001 \\
\hline Locais de procriação & 0,724 & 0,630 & 0,271 & 0,603 & 5,552 & 0,000 & 3,852 & 0,002 & 3,016 & 0,001 \\
\hline Quais as fontes de informações? & 2,536 & 0,020 & 0,036 & 0,850 & 4,086 & 0,000 & 0,515 & 0,765 & 2,041 & 0,003 \\
\hline \multicolumn{11}{|l|}{ Aspectos ambientais } \\
\hline Origem da água de consumo & 0,425 & 0,863 & 0,379 & 0,538 & 0,471 & 0,856 & 0,12 & 0,988 & 1,317 & 0,143 \\
\hline Há estocagem de água? & 0,859 & 0,525 & 0,947 & 0,331 & 1,139 & 0,336 & 1,305 & 0,260 & 1,184 & 0,248 \\
\hline Qual o destino dos dejetos? & 4,933 & 0,000 & 0,268 & 0,605 & 1,026 & 0,411 & 1,999 & 0,077 & 1,329 & 0,136 \\
\hline Qual o destino do lixo? & 0,605 & 0,726 & 1,491 & 0,223 & 0,413 & 0,894 & 0,02 & 1,000 & 0,379 & 0,997 \\
\hline Criadouros potenciais encontrados & 3,263 & 0,004 & 1,133 & 0,288 & 0,784 & 0,601 & 2,707 & 0,020 & 1,096 & 0,342 \\
\hline \multicolumn{11}{|l|}{ Atitudes preventivas em relação a } \\
\hline Vasos & 0,912 & 0,485 & 0,073 & 0,788 & 1,716 & 0,102 & 2,911 & 0,013 & 1,282 & 0,167 \\
\hline Caixa d'água & 0,632 & 0,704 & 0,203 & 0,653 & 0,556 & 0,791 & 0,72 & 0,609 & 0,946 & 0,538 \\
\hline Pneus & 1,808 & 0,095 & 0,121 & 0,728 & 1,591 & 0,135 & 2,525 & 0,028 & 1,021 & 0,436 \\
\hline Garrafas e latas & 0,722 & 0,632 & 0,294 & 0,588 & 1,87 & 0,072 & 1,57 & 0,166 & 1,857 & 0,008 \\
\hline
\end{tabular}

das residências como reservatório permanente e juntamente com outros (tambor, tanque, barril, tonel, depósito de barro), representam boa chance de se transformar em locais ideais para a proliferação de mosquitos, favorecendo a manutenção de sua população 7,25, mesmo em períodos com baixas precipitações. Outra realidade freqüentemente encontrada se refere ao precário estado de conservação das tampas das caixas d'água, por encontrarem-se danificadas, e pelo descaso ou falta de recursos, a sua substituição torna-se difícil. Esses achados estão em consonância com os resultados encontrados por Lenzi et al. 26, Oliveira \& Valla 27 e Forattini \& Brito 25 . Soma-se a isso, a existência de outros reservatórios como garrafas, latas e pneus, dispostos nos arredores das habitações, compondo o lixo doméstico que contribui para agravar o problema, além do hábito das pessoas cultivarem plantas ornamentais, fazendo de vasos de plantas os depósitos mais freqüentes dentro de casa 28.

Considerando-se a situação da coleta de lixo, verificou-se que uma parcela significativa da população informou a regularidade desse serviço. No entanto, observou-se ainda acúmulo de lixo nos quintais das residências e em algumas ruas, principalmente em terrenos baldios, especialmente em áreas de ocupação irregular e condições de vida mais desfavoráveis onde é limitado o alcance das políticas públicas de saneamento 27,29,30.

Quanto à disposição dos dejetos, os que dispõem de rede pública de esgotamento sanitário representam menos da metade da população, coincidindo com dados da média nacional 31 . Entende-se então que medidas como abastecimento de água potável e a coleta regular de lixo são fundamentais para o controle do Ae. aegyp$t i$, e que, fatores contrários como abastecimento de água descontínuo (gerando necessidade de estocagem) e ausência de coleta e destinação adequada do lixo contribuem para ofertar condições propícias para a proliferação dos vetores do dengue, com conseqüente aumento no número de casos. Esses aspectos do serviço público têm uma relação direta com a renda da população, como foi observado por Fabro ${ }^{32}$, 
Costa \& Natal 29 e Pontes 30 , que observaram correlação entre renda e escolaridade e incidência de dengue em Ribeirão Preto e São José do Rio Preto.

As medidas sistemáticas de controle do Ae. aegypti, ao longo das décadas têm apresentado diversas limitações, de ordem operacional e técnica. Com o fim das "campanhas", abandonouse o conceito de erradicação, que previa cobertura de $100 \%$ do alvo perseguido. Passou-se a trabalhar a perspectiva de controle, buscandose uma convivência com o dengue - porém em níveis toleráveis ao homem e monitoradas por contínua vigilância 33. Objetiva-se assim reduzir a densidade vetorial, visando a reduzir níveis de transmissão, de tal forma que a doença não represente mais um problema à saúde da população ${ }^{34}$.

De acordo com Gubler 23 e Nobre et al. 35, as medidas de controle, como a eliminação de criadouros, a aplicação de larvicidas nos depósitos de água de consumo, nebulização com inseticidas (fumacê) não têm alcançado os resultados esperados. Há necessidade de implementação de estratégias mais eficazes, visando principalmente a promover mudanças de hábito da comunidade que favoreçam a manutenção do ambiente doméstico livre do Ae. aegypti.

A população deste estudo mostrou conhecer as medidas recomendadas pelos órgãos públicos responsáveis pelo controle da doença, assim como as mensagens veiculadas pelos meios de comunicação. Tais mensagens teoricamente ajudariam a melhorar a compreensão do problema do dengue.

No entanto, observa-se um descompasso entre o que se conhece a respeito da doença e as práticas desenvolvidas para eliminação de criadouros dos vetores do dengue. A mudança de comportamento e a participação ativa da comunidade são fundamentais para o combate do dengue e outras endemias 15,17,26,36.

Observa-se com preocupação o baixo índice dos que procuram tratamento em Unidades de Saúde, sugerindo que no Município de São Luís, para cada cinco pessoas acometidas pelo dengue, somente uma procurou atendimento, e conseqüentemente foi notificado o caso. Tal procedimento pode conduzir a uma subnotificação e perda de informações, levando os órgãos de saúde a subestimarem a extensão da doença, prejudicando as estratégias de controle, corroborando achados de outros autores 9,21,37,38.

Em síntese, o presente estudo permite-nos concluir que: a comunidade, de uma maneira geral, dispõe de conhecimento sobre dengue, seu vetor e sua prevenção; a população considera esse agravo como um sério problema de saúde pública; de modo geral observou-se que as condições sócio-econômica, sanitária e ambiental da população eram razoáveis, porém nas suas residências existia considerável número de criadouros potenciais, o que favorece sobremaneira a proliferação do Ae. aegypti, constatando-se uma distância entre conhecimento e mudança de comportamento; entedemos que apesar de se constatar pequenas diferenças entre os distritos sanitários no tocante a regularidade de abastecimento de água e na coleta de lixo, não se verificou diferença no nível de infestação do Aedes e da morbidade da doença, sugerindo que tanto o vetor quanto a doença encontram-se amplamente distribuídos no Município de São Luís, posto que verificou-se que o hábito de estocar água persiste na população independente da melhoria do abastecimento 5; as campanhas educativas institucionais e o uso dos meios de comunicação, apesar de eficientes na transmissão de informações, não têm levado a mudanças de comportamento da população quanto ao controle do vetor; torna-se urgente a necessidade de implementação de estratégias de controle, com vistas a minorar o problema; cabe neste momento, aos setores envolvidos no controle da doença, encontrar meios para estimular a comunidade para o seu necessário envolvimento como medida concreta visando ao controle do Ae. aegypti.

\section{Resumo}

Trata-se de uma investigação amostral com 843 entrevistados, realizado no Município de São Luís, Maranhão, Brasil. Avaliou-se o conhecimento da população sobre dengue, assim como suas condições sócio-econômicas, sanitárias e ambientais. Verificaram-se criadouros potenciais em toda a área pesquisada. A mídia ( $T V$, rádio etc.) foi o meio de comunicação mais importante como fonte de informação sobre a doença para a população. No entanto, observou-se uma lacuna

entre o conhecimento e a prática de atitudes voltadas para evitar a proliferação do mosquito do dengue. Os resultados indicam que se deve buscar estratégias que visem a envolver a população de São Luís para uma participação mais efetiva para evitar a proliferação do vetor e, assim, ajudar no controle do dengue.

Dengue; Aedes; Conhecimentos, Atitudes e Práticas em Saúde 


\section{Colaboradores}

V. S. Gonçalves Neto elaborou e desenvolveu o trabalho. S. G. Monteiro colaborou nas análises estatísticas dos dados. A. G. Gonçalves auxiliou na revisão bibliográfica e redação do artigo. J. M. M. Rebêlo colaborou na análise dos dados e em conjunto com os demais autores, na redação do artigo.

\section{Agradecimentos}

À equipe de profissionais da Superintendência de Vigilância Epidemiológica e Sanitária da Secretaria Municipal de Saúde de São Luís, e à bióloga Cristiane Costa Carvalho, pelo apoio para aplicação do questionário.

\section{Referências}

1. Fundação Nacional de Saúde, Ministério da Saúde. Boletim Eletrônico Epidemiológico 2002; ano 2, n. 2.

2. Silveira AC. Dengue: aspectos epidemiológicos e de controle. Rev Soc Bras Med Trop 1998; 31:5-14.

3. Fundação Nacional de Saúde, Ministério da Saúde. Boletim Eletrônico Epidemiológico 2001; ano 1, n. 1 .

4. Secretaria de Vigilância em Saúde, Ministério da Saúde. Vigilância em saúde: dados e indicadores selecionados (2003). Brasília: Secretaria de Vigilância em Saúde, Ministério da Saúde; 2003.

5. Gonçalves Neto VS, Rebêlo JMM. Aspectos epidemiológicos do dengue no Município de São Luís, Maranhão, Brasil, 1997-2002. Cad Saúde Pública 2004; 20:1427-31.

6. Rebêlo JMM, Costa JML, Silva FS, Pereira YNO, Silva JM. Distribuição de Aedes aegypti e do dengue no Estado do Maranhão, Brasil. Cad Saúde Pública 1999; 15:477-86.

7. Penna MLF. Um desafio para a saúde pública brasileira: o controle do dengue. Cad Saúde Pública 2003; 19:305-9.

8. Instituto Brasileiro de Geografia e Estatística. Anuário estatístico do Brasil. Rio de Janeiro: Instituto Brasileiro de Geografia e Estatística; 2002.

9. Vasconcelos PFC, Lima WO, Raposo ML, Rodrigues, SG, Travassos-da-Rosa ES, Moura CMP, et al. Inquérito soro-epidemiológico na Ilha de São Luís durante epidemia de dengue no Maranhão. Rev Soc Bras Med Trop 1999; 32:171-9.

10. Ayres M, Aires Jr. M, Ayres DL, Santos AS. BioEstat 3.0: aplicações estatísticas nas áreas das ciências biológicas e médicas. Belém: Sociedade Civil Mamirauá/Brasília: Conselho Nacional de Desenvolvimento Científico e Tecnológico; 2003.
11. Secretaria Municipal de Saúde do Município de São Luís. Relatório anual de atividades, período: 2002. São Luís: Secretaria Municipal de Saúde do Município de São Luís; 2003.

12. Fundação Nacional de Saúde, Ministério da Saúde. Dengue. Instruções para pessoal de combate ao vetor: manual de normas técnicas. 3a Ed. Brasília: Ministério da Saúde; 2001.

13. Secretaria de Vigilância em Saúde, Ministério da Saúde. Doenças infecciosas e parasitárias: guia de bolso. 3a Ed. Brasília: Secretaria de Vigilância em Saúde, Ministério da Saúde; 2004.

14. Chiaravalloti Neto FC. Conhecimentos da população sobre dengue, seus vetores e medidas de controle em São José do Rio Preto, São Paulo. Cad Saúde Pública 1997; 13:447-53.

15. Chiaravalloti Neto FC, Moraes MS, Fernandes MA. Avaliação dos resultados de atividades de incentivo à participação da comunidade no controle da dengue em um bairro periférico do Município de São José do Rio Preto, São Paulo, e da relação entre conhecimentos e práticas desta população. Cad Saúde Pública 1998; 14 Suppl 2:101-9.

16. Donalísio MR, Alves JCP, Visockas A. Inquérito sobre conhecimentos e atitudes da população sobre a transmissão do dengue - região de Campinas, São Paulo, Brasil - 1998. Rev Soc Bras Med Trop 2001; 34:197-201.

17. Winch P, Lloyd L, Godas MD, Kendall C. Beliefs about the prevention of dengue and other febrile illnesses in Merida, México. J Trop Med Hyg 1991; 94:377-87.

18. Lloyd L, Winch P, Ortega-Canto J, Kendal C. Results of a community-based Aedes aegypti control program in Merida, Yucatan, Mexico. Am J Trop Med Hyg 1992; 46:635-42. 
19. Fajardo P, Monje CA, Lozano G, Realpe O, Hernández LE. Nociones populares sobre "dengue" y "rompehuesos", dos modelos de la enfermedad en Colombia. Rev Panam Salud Publica 2001; 10:161-8.

20. Chiaravalloti VB, Morais MS, Chiaravalloti Neto F, Conversani DT, Fiorin AM, Barbosa AAC, et al. Avaliação sobre a adesão às práticas preventivas do dengue: o caso de Catanduva, São Paulo, Brasil. Cad Saúde Pública 2002; 18:1321-9.

21. Teixeira MG, Barreto ML, Costa MCN, Ferreira LDA, Vasconcelos P. Dinâmica de circulação do vírus da dengue em uma área metropolitana do Brasil. Epidemiol Serv Saúde 2003; 12:87-97.

22. Teixeira MG, Barreto LM, Guerra Z. Epidemiologia e medidas de prevenção do dengue. Inf Epidemiol SUS 1999; 8:5-33.

23. Gubler DJ. Aedes aegypti and Aedes albopictus. Borne disease control in the 1990's: top down or bottom up. Am J Trop Med Hyg 1989; 40:571-8.

24. Organização Pan-Americana da Saúde. Diretrizes relativas à prevenção e ao controle da dengue e da dengue hemorrágica nas Américas. Relatório da reunião sobre diretrizes para a dengue. Washington DC: Organização Pan-Americana da Saúde; 1991.

25. Forattini OP, Brito M. Reservatórios domiciliares de água e controle do Aedes aegypti. Rev Saúde Pública 2003; 37:676-7.

26. Lenzi MF, Camilo-Coura L, Grault CE, Val MB. Estudo do dengue em área urbana favelizada do Rio de Janeiro: considerações iniciais. Cad Saúde Pública 2000; 16:851-6.

27. Oliveira RM, Valla VV. As condições e as experiências de vida de grupos populares no Rio de Janeiro: repensando a mobilização popular no controle do dengue. Cad Saúde Pública 2001; 17 Suppl: 77-88.

28. Moura-Lima M, Aragão MB, Amaral RS. Criadouros de Aedes aegypti encontrados em alguns bairros do Rio de Janeiro, 1984-85. Cad Saúde Pública 1988; 4:293-300.
29. Costa AIP, Natal D. Distribuição espacial da dengue e determinantes socioeconômicos em localidade urbana no sudeste do Brasil. Rev Saúde Pública 1998; 32:232-6.

30. Pontes RJS. Estudo da epidemia de dengue no município de Ribeirão Preto-SP, 1991-1992 [Tese de Doutorado]. Ribeirão Preto: Faculdade de Medicina de Ribeirão Preto, Universidade de São Paulo; 1992.

31. Secretaria de Vigilância em Saúde, Ministério da Saúde. Saúde Brasil 2004: uma análise da situação de saúde. Brasília: Secretaria de Vigilância em Saúde, Ministério da Saúde, 2004.

32. Fabro ALD. Estudo epidemiológico do dengue em Ribeirão Preto no período 1990-1997 [Tese de Doutorado]. Ribeirão Preto: Faculdade de Medicina de Ribeirão Preto, Universidade de São Paulo; 1997.

33. Waldman EA. Vigilância em saúde pública. São Paulo: Instituto para o Desenvolvimento da Saúde/Editora Fundação Peirópolis; 1998.

34. Organización Panamericana de la Salud. Dengue y dengue hemorrágico en las Américas: guías para su prevención y control. Washington DC: Organización Panamericana de la Salud; 1995. (Publicación Científica, 548).

35. Nobre A, Antezana D, Tauil PL. Febre amarela e dengue no Brasil: epidemiologia e controle. Rev Soc Bras Med Trop 1994; 27 Suppl III:59-66.

36. Chor D. Saúde pública e mudanças de comportamento: uma questão contemporânea. Cad Saúde Pública 1999; 15:423-5.

37. World Health Organization. Dengue haemorrhagic fever: diagnosis, treatment and control. 2nd Ed Geneva: World Health Organization; 1997.

38. Pan American Health Organization. Dengue and dengue hemorrhagic fever in the Americas: guidelines for prevention and control. Washington DC: Pan American Health Organization; 1994.

Recebido em 17/Fev/2005

Versão final reapresentada em 17/Fev/2006 Aprovado em 10/Mar/2006 